\title{
Caracterização e avaliação morfológica de frutos em genótipos de pupunheira para
}

\section{mesa}

\author{
Characterization and morphological evaluation of fruits in peach palm genotypes for table \\ Caracterización y evaluación morfológico de frutos en genótipos de pejibaye para mesa
}

Recebido: 30/04/2021 | Revisado: 09/05/2021 | Aceito: 12/05/2021 | Publicado: 29/05/2021

\author{
Camila Pinto Brandão \\ ORCID: https://orcid.org/0000-0003-4658-1253 \\ Universidade Federal Rural da Amazônia, Brasil \\ E-mail: brandaopcamila@gmail.com \\ Maria do Socorro Padilha de Oliveira \\ ORCID: https://orcid.org/0000-0002-4753-2018 \\ Embrapa Amazônia Oriental, Brasil \\ E-mail: socorro-padilha.oliveira@embrapa.br \\ Moisés Cordeiro Mourão de Oliveira Junior \\ ORCID: https://orcid.org/0000-0001-8907-074X \\ Embrapa Amazônia Oriental, Brasil \\ E-mail: mmouraojr@gmail.com
}

\begin{abstract}
Resumo
Na Embrapa Amazônia Oriental, há pupunheiras (Bactris gasipaes Kunth.) de diferentes procedências conservadas no Banco de germoplasma (BAG) em Tomé-Açu (PA). No entanto, pouco se conhece sobre as características morfoagronômicas de seus frutos. $\mathrm{O}$ objetivo do trabalho foi caracterizar e avaliar frutos de genótipos dessa palmeira conservados no BAG visando identificar plantas com características promissoras para frutos de mesa. Foram estudadas 100 pupunheiras representantes de 50 acessos, das quais foram coletadas amostras de 15 frutos maduros de cada planta, as quais foram avaliadas e caracterizadas, morfologicamente, para dez caracteres quantitativos e seis qualitativos. Os dados foram submetidos as análises uni e multivariada (agrupamento) para definição dos grupos mais homogêneos. Em seguida, foi realizada uma concatenação entre os grupos formados pelos caracteres quantitativos e qualitativos. Observou-se divergência dentre os genótipos avaliados, sendo possível a formação de cinco grupos distintos com base nos caracteres quantitativos e oito grupos relacionados aos caracteres qualitativos. A análise conjunta dos caracteres permitiu a identificação de 45 genótipos com características promissoras ao mercado de mesa.

Palavras-chave: Agrupamento; Bactris gasipaes; Caráter; Germoplasma.
\end{abstract}

\begin{abstract}
At Embrapa Amazônia Oriental, there are peach palm (Bactris gasipaes Kunth.) from different sources conserved in the Germplasm Bank (BAG) in Tomé-Açu (PA). However, little is known about the morpho-agronomic characteristics of its fruits. The objective of the work was to characterize and evaluate fruits of genotypes of this palm conserved in the BAG aiming to identify plants with promising characteristics for table fruits. 100 peach palm representing of 50 accessions were studied, from which samples were collected from 15 ripe fruits of each plant, which were evaluated and characterized, morphologically, by ten quantitative and six qualitative characters. The data were subjected to univariate and multivariate analyzes (grouping) to define the most homogeneous groups. Then, there was a concatenation between the groups formed by the quantitative and qualitative characters. There was a divergence among the evaluated genotypes, making it possible to form five distinct groups based on quantitative characters and eight groups related to qualitative characters. The joint character analysis allowed the identification of 45 genotypes with promising characteristics for the table market.
\end{abstract}

Keywords: Bactris gasipaes; Character; Germplasm; Grouping.

\section{Resumen}

En Embrapa Amazônia Oriental, hay pejibaye (Bactris gasipaes Kunth.) de diferentes fuentes conservadas en el Banco de Germoplasma (BAG) en Tomé-Açu (PA). Sin embargo, se sabe poco sobre las características morfoagronómicas de sus frutos. El objetivo del trabajo fue caracterizar y evaluar frutos de genotipos de esta palmera conservados en el BAG con el objetivo de identificar plantas con características promisorias para frutos de mesa. Se estudiaron 100 pejibayes que representan 50 accesos, de las cuales se recolectaron muestras de 15 frutos maduros de cada planta, que fueron evaluados y caracterizados morfológicamente por diez caracteres cuantitativos y seis cualitativos. Los datos fueron sometidos a análisis univariados y multivariados (agrupamiento) para definir los grupos más homogéneos. Luego, cumplido una concatenación entre los grupos formados por los caracteres cuantitativos y 
cualitativos. Se observó una divergencia entre los genotipos evaluados, lo que permitió formar cinco grupos distintos basados en caracteres cuantitativos y ocho grupos relacionados con caracteres cualitativos. El análisis de carácter conjunto permitió identificar 45 genotipos con características prometedoras para el mercado de la mesa.

Palabras clave: Agrupamiento; Bactris gasipaes; Carácter; Germoplasma.

\section{Introdução}

Recursos genéticos vegetais conservados em Bancos de Germoplasma apresentam-se como importantes estratégias para uso em diferentes finalidades na incorporação da variabilidade genética em programas de melhoramento (Burle \& Oliveira, 2010). No caso da pupunheira (Bactris gasipaes Kunth.), são registrados cinco bancos de germoplasma no Brasil (Portal Alelo, 2019), todos conservados em nível de campo em várias localidades, os quais podem atender materiais genéticos para diversas finalidades, tais como a obtenção de material para melhoramento de palmito, frutos para mesa, ração animal, farinha, amido e extração de óleo (Clement et al., 2009). Um deles está na Embrapa Amazônia Oriental e possui 73 acessos, onde 50 deles foram obtidos em feiras livres e representados por 800 genótipos (Alelo Embrapa, 2019; Clement et al., 2009). Entretanto, as atividades de caracterização e avaliação desses genótipos tem sido pouco efetivado, tendo a necessidade de se obter informações desses materiais conservados in vivo.

As atividades de caracterização e avaliação, além de disponibilizar conhecimento do material conservado apresentam outras vantagens, como a identificação de acessos duplicados, o estabelecimento de coleções nucleares, a identificação dos modos de reprodução e, também, de pré-produtos desejáveis a programas de melhoramento genético (Burle, 2019; Oliveira et al., 2019). Além disso, permite quantificar a variação fenotípica existente, no caso da palmeira em foco, frutos de diferentes formatos, tamanhos, composição do mesocarpo, entre outros (Clement et al., 2009).

De acordo com Burle (2019) na caracterização morfoagronômica deve-se considerar descritores de alta herdabilidade, que sejam fáceis de mensurar, além da baixa interação genótipo $\mathrm{x}$ ambiente. $\mathrm{O}$ uso de caracteres morfológicos interpretados com o auxílio de análises multivariadas tem sido muito utilizado por curadores de Bancos de Germoplasma e por melhoristas de plantas (Farias Neto et al., 2020; Oliveira et al., 2019; Santos et al, 2017; Silva et al., 2019)

Vale ressaltar que o Banco de Germoplasma de pupunheira da Embrapa Amazônia Oriental, estudos de caracterização e avaliação têm sido realizados, porém, com poucos avanços (Carvalho et al., 2013; Farias Neto et al., 2013), sendo necessário estudos desta natureza que são subsídios à programas de melhoramento.

O objetivo do trabalho foi caracterizar e avaliar morfologicamente frutos de genótipos de pupunheira, visando identificar genótipos com características promissoras para frutos de mesa.

\section{Metodologia}

Foram amostrados frutos maduros em genótipos de pupunheira, conservados no Banco Ativo de Germoplasma da Embrapa Amazônia Oriental. Esses genótipos foram instalados em uma área experimental de Tomé-açu, PA (2²4’50,83” S, $48^{\circ} 08^{\prime} 59,77^{\prime \prime}$ W). Este município está inserido no Nordeste Paraense, cujo clima é quente e úmido, com precipitação pluviométrica média anual $2.307 \mathrm{~mm}$ e temperatura média anual de $26^{\circ} \mathrm{C}$ (INMET, 2019).

O Banco foi instalado, em 2006, com 50 acessos (progênies de polinização livre), em delineamento de blocos ao acaso - DBC, com 16 blocos e parcela de uma planta, no espaçamento de $6 \mathrm{~m} \mathrm{x} 4 \mathrm{~m}$, totalizando 800 plantas. Para este trabalho a coleta de dados foi feita em dois blocos, ou seja, em duas plantas representantes de cada acesso, totalizando 100 plantas (Figura 1). Na Tabela 1, encontra-se a identificação e procedência dos 100 genótipos, adquiridos em feiras livres de vários municípios de três Estados: Pará, Amazonas e Rondônia. 
Figura 1. Localização geográfica dos 100 genótipos de pupunheira (Bactris gasipaes) conservados no Banco Ativo de Germoplasma da Embrapa Amazônia Oriental, Campo experimental de Tomé-Açu (PA), utilizados no estudo.

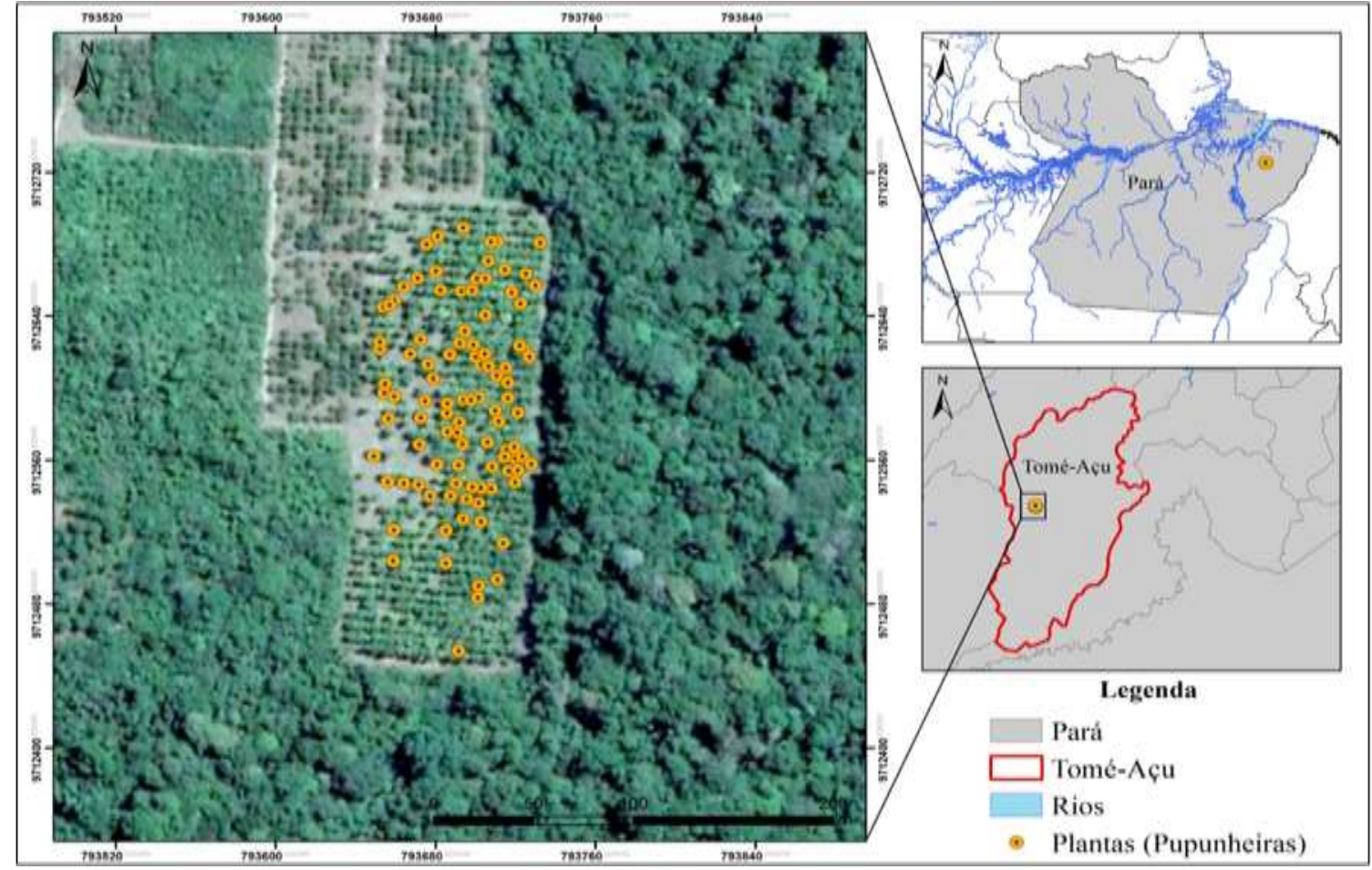

Fonte: Autores.

Tabela 1. Identificação e procedência dos 100 genótipos de pupunheira (B. gasipaes) conservados no Banco Ativo de Germoplasma da Embrapa Amazônia Oriental, Campo experimental de Tomé-Açu (PA).

\begin{tabular}{|c|c|c|c|c|c|}
\hline \multirow{2}{*}{ Genótipos } & \multirow{2}{*}{$\begin{array}{c}\text { Acesso } \\
\text { BAG }\end{array}$} & \multirow{2}{*}{ Procedência } & \multicolumn{3}{|c|}{ Localização geográfica } \\
\hline & & & Latitude & Longitude & Altitude (m) \\
\hline 1 e 2 & 1 & Manicoré (AM) & $05^{\circ} 48^{\prime} 33^{\prime \prime}$ & $61^{\circ} 18^{\prime} 01^{\prime \prime}$ & 45 \\
\hline 3 e 4 & 2 & Manicoré (AM) & $05^{\circ} 48^{\prime} 33^{\prime \prime}$ & $61^{\circ} 18^{\prime} 01^{\prime \prime}$ & 45 \\
\hline 5 e 6 & 3 & Borba (AM) & $04^{\circ} 23^{\prime} 16^{\prime \prime}$ & $59^{\circ} 35^{\prime} 38^{\prime \prime}$ & 45 \\
\hline 7 e 8 & 4 & Borba (AM) & $04^{\circ} 23^{\prime} 16^{\prime \prime}$ & $59^{\circ} 35^{\prime} 38^{\prime \prime}$ & 45 \\
\hline 9 e 10 & 5 & Porto Velho (RO) & $08^{\circ} 45^{\prime} 43^{\prime \prime}$ & $63^{\circ} 54^{\prime} 14^{\prime \prime}$ & 85 \\
\hline 11 e 12 & 6 & Porto Velho (RO) & $08^{\circ} 45^{\prime} 43^{\prime \prime}$ & $63^{\circ} 54^{\prime} 14^{\prime \prime}$ & 85 \\
\hline 13 e 14 & 7 & Humaitá (AM) & $07^{\circ} 30^{\prime} 22^{\prime \prime}$ & $63^{\circ} 01^{\prime} 15^{\prime \prime}$ & 58 \\
\hline 15 e 16 & 8 & Tomé-Açu (PA) & $02^{\circ} 25^{\prime} 08^{\prime \prime}$ & $48^{\circ} 09^{\prime} 08^{\prime \prime}$ & 45 \\
\hline 17 e 18 & 9 & Tomé-Açu (PA) & $02^{\circ} 25^{\prime} 08^{\prime \prime}$ & $48^{\circ} 09^{\prime} 08^{\prime \prime}$ & 45 \\
\hline 19 e 20 & 10 & Santarém (PA) & $02^{\circ} 26^{\prime} 35^{\prime \prime}$ & $54^{\circ} 42^{\prime} 30^{\prime \prime}$ & 51 \\
\hline 21 e 22 & 11 & Sto. Antônio do Tauá (PA) & $01^{\circ} 09^{\prime} 07^{\prime \prime}$ & $48^{\circ} 07^{\prime} 46^{\prime \prime}$ & 17 \\
\hline 23 e 24 & 12 & Colares (PA) & $00^{\circ} 56^{\prime} 12^{\prime \prime}$ & $48^{\circ} 16^{\prime} 54^{\prime \prime}$ & 15 \\
\hline 25 e 26 & 13 & Colares (PA) & $00^{\circ} 56^{\prime} 12^{\prime \prime}$ & $48^{\circ} 16^{\prime} 54^{\prime \prime}$ & 15 \\
\hline 27 e 28 & 14 & Moju (PA) & $01^{\circ} 53^{\prime} 02^{\prime \prime}$ & $48^{\circ} 46^{\prime} 08^{\prime \prime}$ & 16 \\
\hline 29 e 30 & 15 & Moju (PA) & $01^{\circ} 53^{\prime} 02^{\prime \prime}$ & $48^{\circ} 46^{\prime} 08^{\prime \prime}$ & 16 \\
\hline 31 e 32 & 16 & Almeirim (PA) & $01^{\circ} 31^{\prime} 24^{\prime \prime}$ & $52^{\circ} 34^{\prime} 54^{\prime \prime}$ & 65 \\
\hline 33 e 34 & 17 & Almeirim (PA) & $01^{\circ} 31^{\prime} 24^{\prime \prime}$ & $52^{\circ} 34^{\prime} 54^{\prime \prime}$ & 65 \\
\hline 35 e 36 & 18 & Abaetetuba (PA) & $01^{\circ} 43^{\prime} 05^{\prime \prime}$ & $48^{\circ} 52^{\prime} 57^{\prime \prime}$ & 10 \\
\hline 37 e 38 & 19 & Abaetetuba (PA) & $01^{\circ} 43^{\prime} 05^{\prime \prime}$ & $48^{\circ} 52^{\prime} 57^{\prime \prime}$ & 10 \\
\hline 39 e 40 & 20 & Abaetetuba (PA) & $01^{\circ} 43^{\prime} 05^{\prime \prime}$ & $48^{\circ} 52^{\prime} 57^{\prime \prime}$ & 10 \\
\hline 41 e 42 & 21 & Vigia (PA) & $00^{\circ} 51^{\prime} 30^{\prime \prime}$ & $48^{\circ} 08^{\prime} 30^{\prime \prime}$ & 6 \\
\hline 43 e 44 & 22 & Vigia (PA) & $00^{\circ} 51^{\prime} 30^{\prime \prime}$ & $48^{\circ} 08^{\prime} 30^{\prime \prime}$ & 6 \\
\hline 45 e 46 & 23 & Vigia (PA) & $00^{\circ} 51^{\prime} 30^{\prime \prime}$ & $48^{\circ} 08^{\prime} 30^{\prime \prime}$ & 6 \\
\hline 47 e 48 & 24 & Vigia (PA) & $00^{\circ} 51^{\prime} 30^{\prime \prime}$ & $48^{\circ} 08^{\prime} 30^{\prime \prime}$ & 6 \\
\hline 49 e 50 & 25 & Acará (PA) & $01^{\circ} 57^{\prime} 39^{\prime \prime}$ & $48^{\circ} 11^{\prime} 48^{\prime \prime}$ & 25 \\
\hline 51 e 52 & 26 & Acará (PA) & $01^{\circ} 57^{\prime} 39^{\prime \prime}$ & $48^{\circ} 11^{\prime} 48^{\prime \prime}$ & 25 \\
\hline
\end{tabular}




\begin{tabular}{|c|c|c|c|c|c|}
\hline 53 e 54 & 27 & Acará (PA) & $01^{\circ} 57^{\prime} 39^{\prime \prime}$ & $48^{\circ} 11^{\prime} 48^{\prime \prime}$ & 25 \\
\hline 55 e 56 & 28 & Acará (PA) & $01^{\circ} 57^{\prime} 39^{\prime \prime}$ & $48^{\circ} 11^{\prime} 48^{\prime \prime}$ & 25 \\
\hline 57 e 58 & 29 & Acará (PA) & $01^{\circ} 57^{\prime} 39^{\prime \prime}$ & $48^{\circ} 11^{\prime} 48^{\prime \prime}$ & 25 \\
\hline 59 e 60 & 30 & Concórdia do Pará (PA) & $02^{\circ} 00^{\prime} 06^{\prime \prime}$ & $47^{\circ} 56^{\prime} 59^{\prime \prime}$ & 440 \\
\hline 61 e 62 & 31 & Concórdia do Pará (PA) & $02^{\circ} 00^{\prime} 06^{\prime \prime}$ & $47^{\circ} 56^{\prime} 59^{\prime \prime}$ & 440 \\
\hline 63 e 64 & 32 & Bujarú (PA) & $01^{\circ} 30^{\prime} 54^{\prime \prime}$ & $48^{\circ} 02^{\prime} 41^{\prime \prime}$ & 10 \\
\hline 65 e 66 & 33 & Bujarú (PA) & $01^{\circ} 30^{\prime} 54^{\prime \prime}$ & $48^{\circ} 02^{\prime} 41^{\prime \prime}$ & 10 \\
\hline 67 e 68 & 34 & Bujarú (PA) & $01^{\circ} 30^{\prime} 54^{\prime \prime}$ & $48^{\circ} 02^{\prime} 41^{\prime \prime}$ & 10 \\
\hline 69 e 70 & 35 & Bujarú (PA) & $01^{\circ} 30^{\prime} 54^{\prime \prime}$ & $48^{\circ} 02^{\prime} 41^{\prime \prime}$ & 10 \\
\hline 71 e 72 & 36 & Bujarú (PA) & $01^{\circ} 30^{\prime} 54^{\prime \prime}$ & $48^{\circ} 02^{\prime} 41^{\prime \prime}$ & 10 \\
\hline 73 e 74 & 37 & Sta. Izabel do Pará (PA) & $01^{\circ} 17^{\prime} 55^{\prime \prime}$ & $48^{\circ} 09^{\prime} 38^{\prime \prime}$ & 24 \\
\hline 75 e 76 & 38 & Sta. Izabel do Pará (PA) & $01^{\circ} 17^{\prime} 55^{\prime \prime}$ & $48^{\circ} 09^{\prime} 38^{\prime \prime}$ & 24 \\
\hline 77 e 78 & 39 & Belém (PA) & $01^{\circ} 27^{\prime} 21^{\prime \prime}$ & $48^{\circ} 30^{\prime} 16^{\prime \prime}$ & 10 \\
\hline 79 e 80 & 40 & Belém (PA) & $01^{\circ} 27^{\prime} 21^{\prime \prime}$ & $48^{\circ} 30^{\prime} 16^{\prime \prime}$ & 10 \\
\hline 81 e 82 & 41 & Belém (PA) & $01^{\circ} 27^{\prime} 21^{\prime \prime}$ & $48^{\circ} 30^{\prime} 16^{\prime \prime}$ & 10 \\
\hline 83 e 84 & 42 & Belém (PA) & $01^{\circ} 27^{\prime} 21^{\prime \prime}$ & $48^{\circ} 30^{\prime} 16^{\prime \prime}$ & 10 \\
\hline 85 e 86 & 43 & Belém (PA) & $01^{\circ} 27^{\prime} 21^{\prime \prime}$ & $48^{\circ} 30^{\prime} 16^{\prime \prime}$ & 10 \\
\hline 87 e 88 & 44 & Belém (PA) & $01^{\circ} 27^{\prime} 21^{\prime \prime}$ & $48^{\circ} 30^{\prime} 16^{\prime \prime}$ & 10 \\
\hline 89 e 90 & 45 & Belém (PA) & $01^{\circ} 27^{\prime} 21^{\prime \prime}$ & $48^{\circ} 30^{\prime} 16^{\prime \prime}$ & 10 \\
\hline 91 e 92 & 46 & Belém (PA) & $01^{\circ} 27^{\prime} 21^{\prime \prime}$ & $48^{\circ} 30^{\prime} 16^{\prime \prime}$ & 10 \\
\hline 93 e 94 & 47 & Belém (PA) & $01^{\circ} 27^{\prime} 21^{\prime \prime}$ & $48^{\circ} 30^{\prime} 16^{\prime \prime}$ & 10 \\
\hline 95 e 96 & 48 & Belém (PA) & $01^{\circ} 27^{\prime} 21^{\prime \prime}$ & $48^{\circ} 30^{\prime} 16^{\prime \prime}$ & 10 \\
\hline 97 e 98 & 49 & Belém (PA) & $01^{\circ} 27^{\prime} 21^{\prime \prime}$ & $48^{\circ} 30^{\prime} 16^{\prime \prime}$ & 10 \\
\hline 99 e 100 & 50 & Belém (PA) & $01^{\circ} 27^{\prime} 21^{\prime \prime}$ & $48^{\circ} 30^{\prime} 16^{\prime \prime}$ & 10 \\
\hline
\end{tabular}

Fonte: Portal Alelo (2019).

O presente estudo seguiu a metodologia de avaliação e caracterização de frutos proposta de Clement (1986) com adaptações no número de amostras descritas a seguir. De cada genótipo foram colhidos três cachos consecutivos, sendo retirada uma amostra de cinco frutos ao acaso por cacho, totalizando 15 frutos por genótipo. Após a colheita as amostras foram identificadas, acondicionadas em sacos de plástico e transportadas até o Laboratório de Fitomelhoramento na sede dessa instituição, em Belém, PA.

Cada amostra foi avaliada e caracterizada, morfologicamente, para dez caracteres quantitativos e seis qualitativos, num total de 16 caracteres, com base na lista de descritores mínimos elaborada para a pupunha por Clement (1986), quais sejam:

Caracteres quantitativos: diâmetro longitudinal (DL - mm), diâmetro transversal (DT - mm), espessura da polpa (EP $\mathrm{mm})$, espessura do endocarpo (EE - mm), espessura da amêndoa (EA - mm) e o comprimento da semente (CS - mm) foram mensurados com auxílio de paquímetro. Pesos do fruto (PF - g), peso da polpa (PP - g) e peso da semente (PS - g), mensurados com auxílio de balança de precisão (Figura 2). O rendimento de polpa por fruto (RPF - \%) foi obtido pela razão entre os caracteres ((PP/PF) e multiplicado por 100). 
Figura 2. Caracteres quantitativos mensurados nas amostras de frutos de pupunheira, com base na lista de descritores de Clement $(1986)^{*}$.
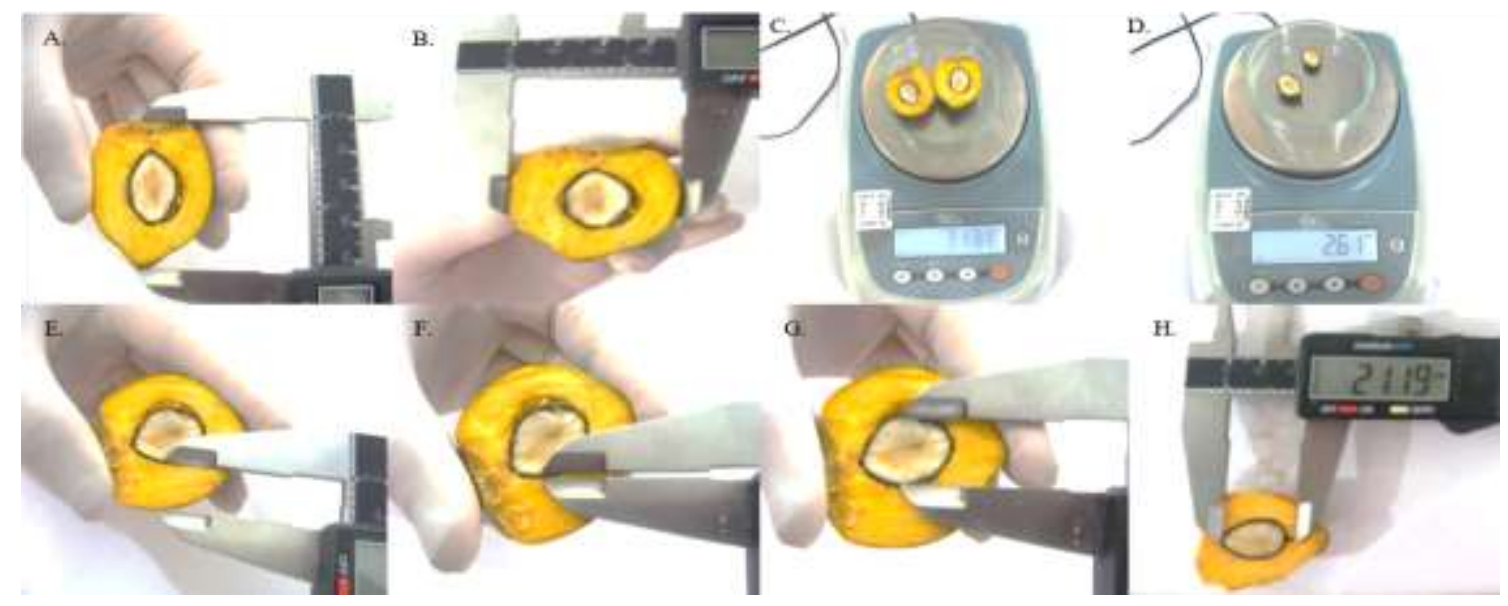

* Diâmetro longitudinal (A.), diâmetro transversal (B.), peso do fruto (C.), peso da semente (D.), espessura da polpa (E.), espessura do endocarpo (F.), espessura da amêndoa (G.) e comprimento da semente (H.).

Fonte: Autores.

Caracteres qualitativos: ocorrência de partenocarpia (OPART), ocorrência de praga (OP), ocorrência de doença (OD) e ocorrência de rachadura (OR), em escala de notas sendo " 0 " para ausência e "1" para presença (Figuras 3A; 3B; 3C e 3D). A cor da casca (CorC) e da polpa (CorP) foi feita com o auxílio da carta de cores para vegetal (Munsell, 1977) (Figuras 3E e 3F).

Figura 3. Caracteres qualitativos observados em amostras de frutos de pupunheira, com base na lista de descritores de Clement $(1986)^{*}$
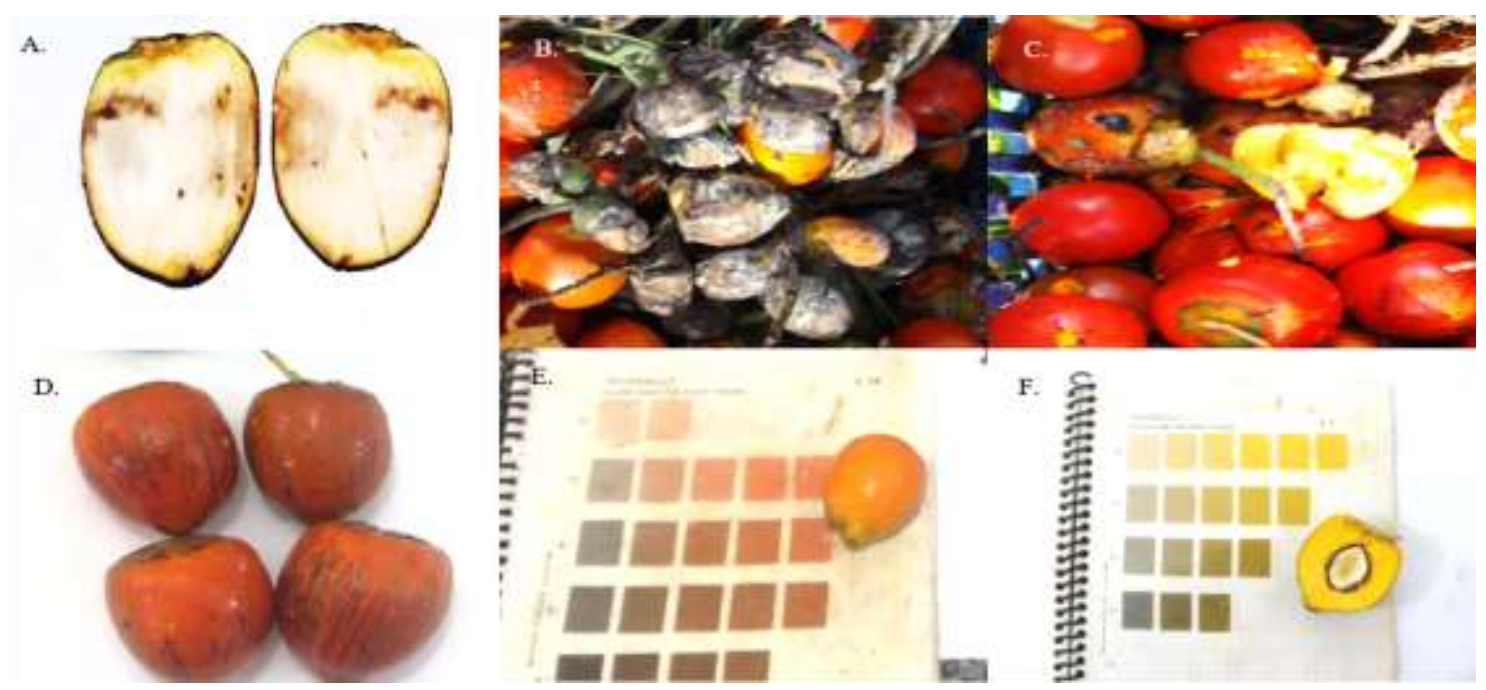

*Ocorrência de partenocarpia (A.), ocorrência de doença, (B.), ocorrência de praga (C.), ocorrência de rachadura (D.), cor da casca (F.) e cor da polpa (G.).

Fonte: Autores.

Os caracteres quantitativos e qualitativos foram submetidos a uma análise de agrupamento (cluster analysis), com o objetivo de definir grupos mais homogêneos. Sendo utilizada a distância euclidiana padronizada e o método de amalgamação de ligação completa, com auxílio do software R-Program (R Core Team, 2014). Quanto as cores, considerando sua variabilidade, tanto da casca quanto da polpa dos frutos, foram agrupadas em "tons", tais sejam: esverdeado, amarelado, alaranjado e avermelhado para cor da casca e, creme, rosada creme, alaranjada e amarelada para cor da polpa. Essa medida foi utilizada afim de também possibilitar a evidenciação de grupos mais homogêneos. 
Após a definição dos grupos homogêneos, estes foram caracterizados em seus valores médios. Uma concatenação entre os grupos homogêneos obtidos para os caracteres quantitativos e qualitativos foi realizada, a fim de definir uma "tipificação" de frutos dos diferentes acessos de pupunha.

\section{Resultados e Discussão}

A análise de agrupamento, com base nos dez caracteres quantitativos, utilizando-se $50 \%$ de similaridade como critério para definição de grupos, permitiu a formação de cinco grupos distintos (Figura 4). Em ordem decrescente tem-se o grupo "e" que abrangeu 23 genótipos; o grupo "d" com 33 genótipos; o grupo "c" por 31 genótipos, o grupo "b" com 9 genótipos; e grupo "a" por 4 genótipos.

Figura 4. Dendrograma de similaridade entre 100 genótipos de pupunheira (Bactris gasipaes) do BAG da Embrapa Amazônia Oriental, área Tomé Açu, PA, obtida de dez caracteres quantitativos.

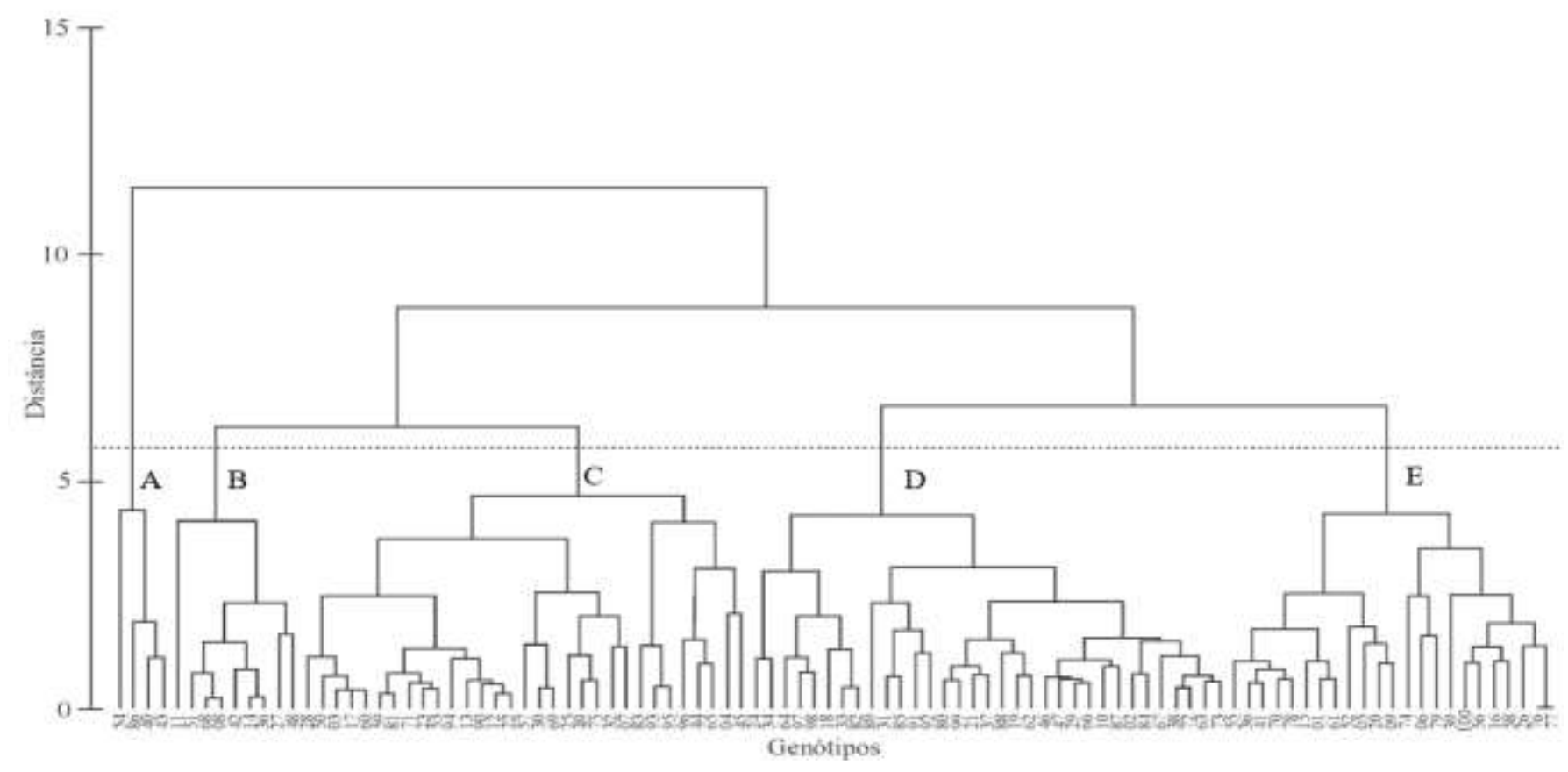

Fonte: Autores.

Cada grupo formado apresentou as seguintes características, conforme as médias obtidas dentre os dez caracteres quantitativo (Tabela 3). Os grupos "a", "d" e "e" expressaram características de frutos de classificação mesocarpa, por apresentarem pesos variando de 20-70 g, com os genótipos do grupo "a", tendo mais afinidade para macrocarpa (peso do fruto acima de $70 \mathrm{~g}$ ) de acordo com a classificação descrita por Mora-Urpí \& Clement (1988), resultados semelhantes foram encontrados por Aguiar et al. (2019) ao avaliarem frutos de acessos de pupunheira tendo frutos com pesos variando de 19,66 g a 81,88g. Em contrapartida, os genótipos formados no grupo "b", caracterizam predominantemente por frutos partenocárpicos, ou seja, sem sementes.

A partenocarpia na pupunheira é um fenômeno muito comum, entretanto, desconhece-se o fator ou fatores determinantes a sua ocorrência. Kalil Filho et al. (2010) sugeriram que essa característica pode estar relacionada com a baix a quantidade de pólen, problemas nutricionais ou deficiência hídrica nas fases de floração e fertilização. Borges (2016) apontou que a autofecundação por incompatibilidade dos gametas pode estar relacionada na ocorrência de frutos partenocárpicos. 
Tabela 3. Características dos grupos formados, com base nos dez caracteres quantitativos, obtido do dendrograma gerado pela distância euclidiana (50\% de similaridade).

\begin{tabular}{|c|c|c|c|c|c|c|}
\hline Grupo & $\begin{array}{c}\text { Genótipos } \\
\left(\mathbf{n}^{\circ}\right)\end{array}$ & $\begin{array}{c}\text { Formato do } \\
\text { fruto (DL-DT) }\end{array}$ & $\begin{array}{c}\text { Peso } \\
\text { do } \\
\text { fruto } \\
(\mathrm{g}) \\
\end{array}$ & Classificação * & $\begin{array}{c}\text { Tamanho da } \\
\text { semente }\end{array}$ & $\begin{array}{c}\text { Peso da } \\
\text { semente } \\
\text { (g) }\end{array}$ \\
\hline A & 4 & $\begin{array}{l}\text { Redondo } \\
(43-44 \mathrm{~mm})\end{array}$ & 61 & mesocarpa & $\begin{array}{l}\text { médio a } \\
\text { grande } \\
(18 \mathrm{~mm})\end{array}$ & 3 \\
\hline B & 9 & $\begin{array}{c}\text { Elíptico } \\
\text { (24-29 mm) }\end{array}$ & 12 & fruto infértil & - & - \\
\hline $\mathrm{C}$ & 31 & $\begin{array}{c}\text { oblongo } \\
(30-33 \mathrm{~mm})\end{array}$ & 20 & microcarpa & $\begin{array}{l}\text { pequena } \\
(2 \mathrm{~mm})\end{array}$ & 0,34 \\
\hline $\mathrm{D}$ & 33 & $\begin{array}{c}\text { redondo } \\
(31-31 \mathrm{~mm})\end{array}$ & 22 & mesocarpa & $\begin{array}{l}\text { médio } \\
(16 \mathrm{~mm})\end{array}$ & 2 \\
\hline $\mathrm{E}$ & 23 & $\begin{array}{c}\text { redondo } \\
(36-36 \mathrm{~mm})\end{array}$ & 33 & mesocarpa & $\begin{array}{l}\text { médio } \\
(17 \mathrm{~mm})\end{array}$ & 3 \\
\hline
\end{tabular}

*Microcarpa (inferior a 20g), mesocarpa (20-70 g) e macrocarpa (acima de $70 \mathrm{~g}$ ) com base a classificação dos autores Mora Urpi e Clement (1988). DL: diâmetro lateral, DT: diâmetro transversal.

Fonte: Autores.

A dissimilaridade entre os genótipos era esperada, devido aos diferentes locais de coleta para a formação do BAG, tendo consequentemente a variabilidade do material coletado. Outro fator a ser considerado é a polinização livre e pelo fato de a pupunheira ser caracterizada como alógama, cujo o modo de reprodução se dar por fecundação cruzada, o que pode contribuir na variabilidade genética na espécie (Kalil Filho et al., 2010).

De modo geral, $60 \%$ dos genótipos de pupunheira avaliados neste estudo, com base nos caracteres quantitativos, mostraram tendência para frutos de formato redondo, de peso médio (mesocarpa) e com sementes de tamanho e peso médio.

O dendrograma gerado para os seis caracteres qualitativos, utilizando-se $50 \%$ de similaridade como critério de definição separou oito grupos, caracterizados como bastante heterogêneos (Figura 5). Em ordem decrescente têm-se: grupo "h" formado por 38 genótipos; o grupo "g” por 2 genótipos; o grupo "f” por 11 genótipos; grupo "e" por 13 genótipos; o grupo "d" por 14 genótipos; o grupo "c" por 7 genótipos; o grupo "b” por 12 genótipos; e o grupo "a" por 3 genótipos. 
Figura 5. Dendrograma de similaridade entre 100 genótipos de pupunheira (Bactris gasipes) do BAG da Embrapa Amazônia Oriental, área Tomé Açu, PA, obtida de dez caracteres qualitativos.

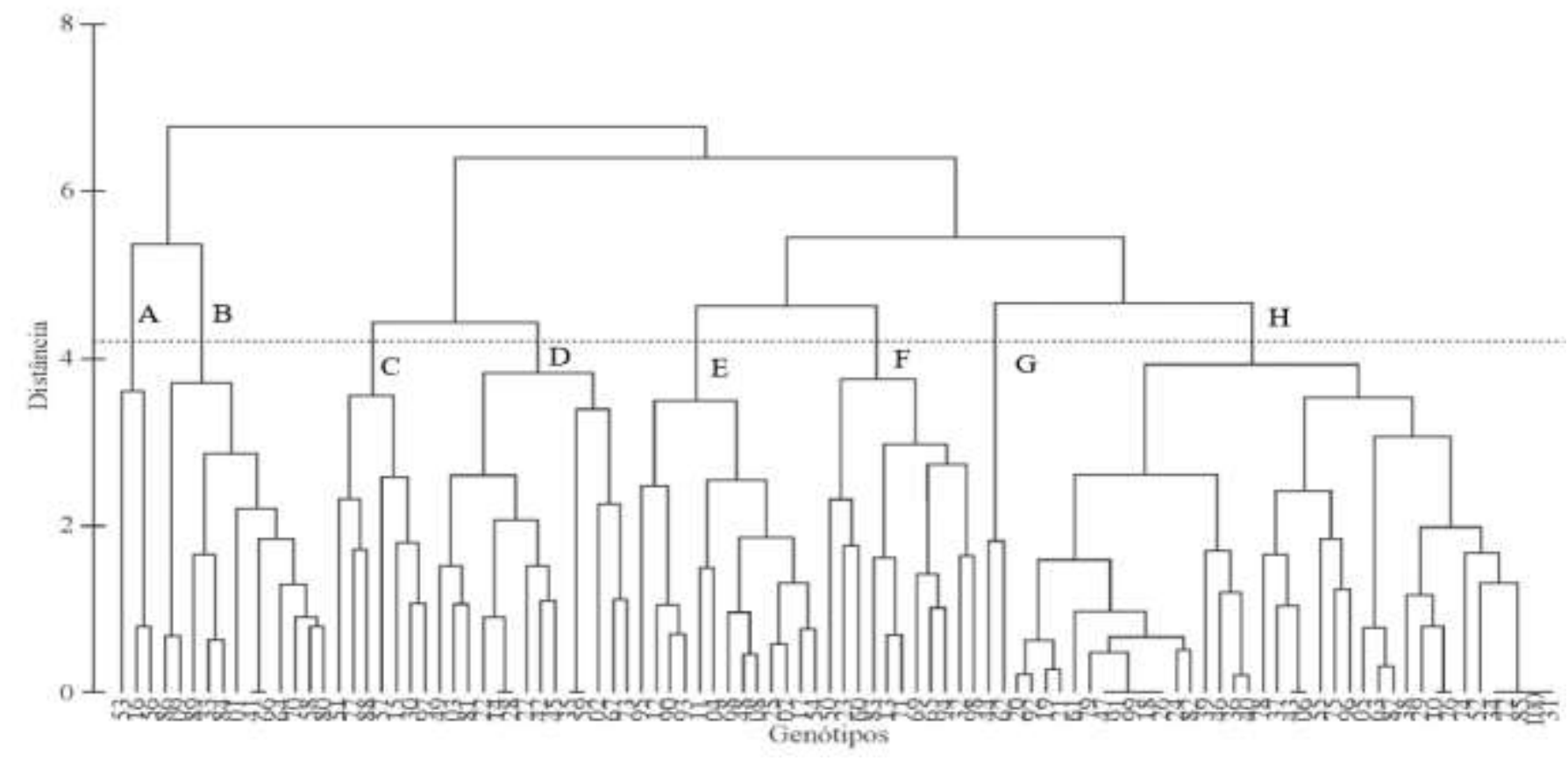

Fonte: Autores.

Observou-se que as características analisadas para os grupos formados foram bastante variáveis, especialmente no que corresponde a cor da casca e da polpa. No entanto, verificou-se que para cor de casca os frutos apresentaram "tons" alaranjado, seguida do amarelo e vermelho e, para a cor da polpa os "tons" mostraram-se mais claros (creme) variando do rosado ao laranja (Tabela 4). Conforme Cornelius et al. (2010) a cor da polpa está associado quanto ao teor de carotenoide, enquanto a cor da casca está relacionado a origem genética. Deste modo o que corresponde na variação da cor do mesocarpo e exocarpo dos frutos avaliados. 
Tabela 4. Características dos grupos formados, com base nos seis caracteres qualitativos, obtido do dendrograma gerado pela distância euclidiana (50\% de similaridade).

\begin{tabular}{|c|c|c|c|c|c|c|c|}
\hline Grupo & $\begin{array}{c}\text { Genótipos } \\
\left(\mathbf{n}^{\circ}\right)\end{array}$ & $\begin{array}{c}\text { Cor } \\
\text { da casca }\end{array}$ & $\begin{array}{c}\text { Cor } \\
\text { da polpa }\end{array}$ & $\begin{array}{c}\text { OPART } \\
(\%)\end{array}$ & $\begin{array}{l}\text { OP } \\
(\%)\end{array}$ & $\begin{array}{l}\text { OD } \\
(\%)\end{array}$ & $\begin{array}{l}\text { OR } \\
(\%)\end{array}$ \\
\hline A & 3 & $\begin{array}{c}\text { Laranja ao } \\
\text { vermelho }\end{array}$ & $\begin{array}{c}\text { Creme ao } \\
\text { laranja }\end{array}$ & 17 & 20 & 50 & 0 \\
\hline B & 12 & $\begin{array}{c}\text { Laranja ao } \\
\text { vermelho }\end{array}$ & $\begin{array}{l}\text { Creme } \\
\text { rosada }\end{array}$ & 5 & 17 & 4 & 5 \\
\hline $\mathrm{C}$ & 7 & $\begin{array}{c}\text { Verde } \\
\text { amarelo }\end{array}$ & Creme & 24 & 30 & 35 & 0 \\
\hline $\mathrm{D}$ & 14 & $\begin{array}{c}\text { Verde } \\
\text { amarelo }\end{array}$ & $\begin{array}{c}\text { Creme ao } \\
\text { amarelo }\end{array}$ & 38 & 29 & 6 & 0 \\
\hline $\mathrm{E}$ & 13 & Amarelo & Creme & 45 & 27 & 11 & 1 \\
\hline $\mathrm{F}$ & 11 & $\begin{array}{l}\text { Amarelo } \\
\text { ao laranja }\end{array}$ & Creme & 41 & 34 & 23 & 3 \\
\hline G & 2 & $\begin{array}{l}\text { Amarelo } \\
\text { ao laranja }\end{array}$ & $\begin{array}{c}\text { Creme ao } \\
\text { laranja }\end{array}$ & 13 & 0 & 40 & 0 \\
\hline $\mathrm{H}$ & 38 & Laranja & $\begin{array}{l}\text { Creme } \\
\text { rosado }\end{array}$ & 3 & 14 & 3 & 0 \\
\hline
\end{tabular}

Fonte: Autores.

Em se tratando de frutos sem sementes esses ocorreram nos oito grupos formados, com destaque para os grupos "d", "e" e "f" que apresentaram forte presença de partenocarpia.

A ocorrência de praga foi registrada em sete grupos, com ênfase para os grupos "c", "d", "e" e "f". Apenas o grupo "g" sem registro de praga.

Em relação à ocorrência de doença três grupos registraram altos percentuais, sugerindo que os genótipos dos grupos “a", "c" e "g" são mais suscetíveis. Uma das principais doenças de importância na cultura da pupunha é a antracnose, causada pelo fungo Colletotrichum gloeosporioides, ocasionando aparecimento de manchas negras em mudas, plantas reprodutivas e nos frutos (Moraes et al., 2017). As principais pragas ocorrentes nessa espécie são causadas por coleópteros Rhynchophorus palmarum e o Metamasius hemipterus (Vaz, 2010). Vale ressaltar que neste estudo não foi realizado levantamento de qual ou quais doenças e pragas presentes nos frutos dos genótipos avaliados.

No caso da ocorrência de rachaduras foram constatados registros em apenas três grupos "b", "e" e "f", porém, com baixo percentual.

Com base nos caracteres qualitativos avaliados nos 100 genótipos de pupunheira pode-se considerar que, $51 \%$ deles possuem predisposição para apresentar cor da casca alaranjado, 34\% amarelo e 15\% de tonalidade avermelhada. Enquanto que para a cor da polpa a coloração creme foi recorrente em todos os genótipos, com variações para "tons" rosado, amarelo e alaranjado.

Com o propósito da definição de tipos de frutos de pupunha ao mercado de mesa, os grupos obtidos com os 100 genótipos receberam classificação "A" para caracteres quantitativos, com três níveis de descrição e, "B" para caracteres qualitativos, com quatro níveis de descrição (Tabela 5). 
Tabela 5. Descrição dos grupos obtidos nos dendrogramas para caracteres quantitativas e qualitativas.

\begin{tabular}{|c|c|}
\hline Grupo A & Descrição \\
\hline A1 & $\begin{array}{l}\text { Frutos médios a grandes }(61 \mathrm{~g}) \\
\text { Formato redondo } \\
\text { Sementes de tamanho médio a grande e pesadas }\end{array}$ \\
\hline $\mathrm{A} 2$ & $\begin{array}{l}\text { Frutos pequenos ( } 12 \mathrm{~g} \text { a } 20 \mathrm{~g}) \\
\text { Formato oblongo } \\
\text { Sementes pequenas e pouco pesadas }\end{array}$ \\
\hline A3 & $\begin{array}{l}\text { Frutos de tamanho médio ( } 22 \mathrm{~g} \text { a } 33 \mathrm{~g}) \\
\text { Formato redondo } \\
\text { Sementes de tamanho médios a grandes e pouco } \\
\text { pesadas }\end{array}$ \\
\hline Grupo B & Descrição \\
\hline B1 & $\begin{array}{l}\text { Frutos de coloração alaranjado } \\
\text { Polpa creme ou alaranjada } \\
\text { Baixa ocorrência de partenocarpia } \\
\text { Baixa ocorrência de praga } \\
\text { Alta ocorrência de doença } \\
\text { Ausência de rachadura }\end{array}$ \\
\hline B2 & $\begin{array}{l}\text { Frutos de coloração alaranjado ao vermelhado } \\
\text { Polpa rosado creme } \\
\text { Baixa ocorrência de partenocarpia } \\
\text { Baixa ocorrência de praga } \\
\text { Baixa ocorrência de doença } \\
\text { Baixa ocorrência de rachadura }\end{array}$ \\
\hline B3 & $\begin{array}{l}\text { Frutos de coloração verde ao amarelo } \\
\text { Polpa creme ou amarelo } \\
\text { Baixa ocorrência de partenocarpia } \\
\text { Baixa ocorrência de praga } \\
\text { Baixa ocorrência de doença } \\
\text { Ausência de rachadura }\end{array}$ \\
\hline B4 & $\begin{array}{l}\text { Frutos de coloração amarelo } \\
\text { Polpa creme } \\
\text { Alta ocorrência de partenocarpia } \\
\text { Baixa ocorrência de praga } \\
\text { Baixa ocorrência de doença } \\
\text { Baixa ocorrência de rachadura }\end{array}$ \\
\hline
\end{tabular}

Fonte: Autores.

A partir da junção dos grupos homogêneos obtidos nos dendrogramas obtidos pelos caracteres quantitativos e qualitativos, foi possível obter a formação de três grandes grupos (Figura 6). Observou-se uma distribuição heterogênea entre os genótipos, e com características distintas entre grupos, com indicativo de existência de variabilidade de frutos no tamanho, no formato, na cor de casca, na cor de polpa e da semente.

Esta heterogeneidade pode estar relacionada a forma de obtenção do material conservado, no caso oriundo de diferentes locais de coletas (diversas feiras-livres) do Nordeste Paraense e ao longo do rio Madeira, no Amazonas. Muitos estudos fizeram abordagem da distribuição das raças de pupunha ao longo dos rios Solimões e Amazonas, através de técnicas moleculares com o uso de marcadores RAPD, os quais validaram a existência das raças Putumayo, Pará, Solimões, Pampa Hermosa, Juruá e Vaupés, além de populações híbridas Belém, Manaus, Iquitos e Yurimaguas (Cristo-Araújo et al., 2010; Rodrigues et al., 2005; Sousa et al., 2001). Podendo-se inferir que os genótipos estudados devem ser constituídos por diferentes raças, refletindo na existência da variabilidade genética.

Com relação aos grupos formados (Figura 6), em ordem decrescente tem-se o grupo A3 composto por 56 genótipos; grupo A2 com 40 genótipos; e grupo A1 com quatro genótipos. Esses grupos, com base nos caracteres avaliados podem ser descritos da seguinte forma: 
Grupo A1: formado por genótipos que possuem frutos médios a grande, com peso médio de $61 \mathrm{~g}$, classificado como mesocarpa (20 -70g), de formato redondo, contendo sementes médias a grandes e pesadas. Os 4 genótipos estão distribuídos em dois níveis para os caracteres qualitativos, sendo, três genótipos no "B2" e um genótipo no "B4". No entanto, predominantemente, o grupo é formado por frutos de coloração da casca variando do laranja ao vermelho, de polpa rosadacreme, com baixas ocorrências de partenocarpia, praga, doença e rachadura.

Grupo A2: constituído por genótipos com frutos pequenos, com peso variando de $12 \mathrm{~g}$ a $20 \mathrm{~g}$, classificados como microcarpa, de formato oblongo, contendo sementes pequenas e pouco pesadas. Os 40 genótipos agregam-se em quatro níveis descritivos para os caracteres qualitativos: "B1" e "B2" com dois genótipos cada, enquanto o "B3" e o "B4" possuem 14 e 22 genótipos, respectivamente. No maior conjunto de genótipos (B4), os frutos possuem coloração da casca amarelada, de polpa creme, com altas ocorrências de partenocarpia, porém, com baixas ocorrências de praga, doença e rachadura.

Grupo A3: contém genótipos cujos frutos são médios, com peso variando de 22g a 33g, classificado como mesocarpa, de formato redondo, contendo sementes médias a grandes e pouco pesadas. Nele os 56 genótipos estão organizados em quatro níveis, com base nos caracteres qualitativos: B4 (1), B1 (3), B3 (7) e B2 (45). No nível B2 os 45 genótipos apresentam frutos de coloração da casca variando do laranja ao vermelho, de polpa rosada-creme, com baixas ocorrências de partenocarpia, praga, doença e rachadura.

Figura 6. Ordenação multidimensional não-métrica (NMDS) dos 100 genótipos de pupunheira (Bactris gasipaes), obtida a partir das matrizes de similaridade euclidiana dos caracteres quantitativos, representando a concatenação entre os agrupamentos a partir da junção das variáveis quantitativas e qualitativas.

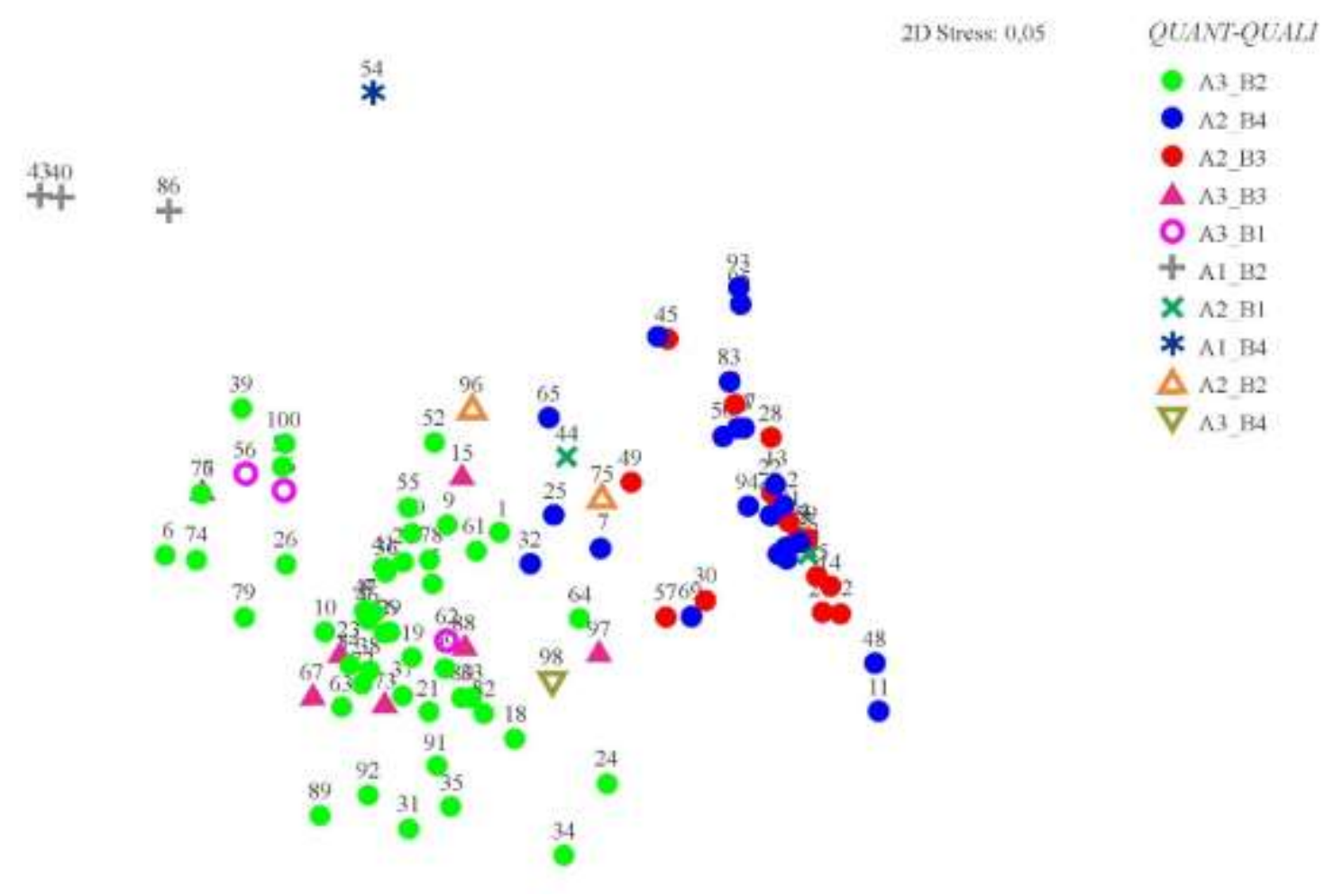

Fonte: Autores.

Conforme Clement et al. (2004) os frutos de pupunha para mesa preferíveis por consumidores são de tamanho médios a grande de coloração avermelhada e oleosos. Deste modo pode-se considerar que os 45 genótipos pertencentes ao nível "B2" do grupo "A3" se mostram promissores, pois são detentores das características físicas e qualitativas apontadas pelos 
consumidores como desejáveis ao mercado de mesa.

\section{Conclusão}

Fatores clássicos na segregação de progênies de pupunheira, tais: partenocarpia e tamanho do fruto ainda constituem uma marcação válida. Uma elevada divergência entre os acessos foi assinalada, mas também evidenciada homogeneidade em vários agrupamentos, quando considerados os caracteres quantitativos e qualitativos. Ressalta-se a existência de um elevado número de progênies avaliadas (45 genótipos) com características promissoras ao mercado de frutos de mesa.

Para melhor conhecimento destes genótipos, sugere-se estudos de avaliação química para obtenção de informação de teor de óleo, fibra e carotenoides totais.

\section{Agradecimentos}

A Fundação Amazônia de Amparo a Estudos e Pesquisas (FAPESPA) pelo fomento à pesquisa, a Coordenação de Pós-graduação em Biotecnologia Aplicada à Agropecuária (PPGBAA - UFRA) e a Embrapa Amazônia Oriental pelo espaço concedido para realização do experimento.

\section{Referências}

Aguiar, J. P. L., Yuyama, K., \& Souza, F. das C. do A. (2019). Caracterização dos frutos de Pupunheira (Bactris gasipaes Kunth) cultivada na vila do Equador, RR: O que há de novo? Scientia Amazonia, 8 (1), 1-5. https://www.researchgate.net/publication/331736505.

Borges, C. V. (2016). Avaliações biométricas de caracteres agronômico em progênies de pupunha (Bactrs gasipaes). Tese (Doutorado em Agronomia Tropical) - Universidade Federal do Amazonas, Manaus, Amazonas, Brasil. https://tede.ufam.edu.br/handle/tede/5458.

Burle, M. L. (2019). Conservação de recursos genéticos vegetais na Embrapa - histórico e perspectivas futuras. Brasília, DF: Embrapa Recursos Genéticos e Biotecnologia, 14 p. (Embrapa Recursos Genéticos e Biotecnologia. Comunicado técnico, http://www.infoteca.cnptia.embrapa.br/infoteca/handle/doc/1108787.

Burle, M. L. \& Oliveira, M. do S. P. de. (2010). Manual de curadores de Germoplasma - Vegetal: caracterização morfológica. Brasília, DF: Embrapa Recursos Genéticos e Biotecnologia; Belém, PA: Embrapa Amazônia Oriental, 15 p. (Embrapa Recursos Genéticos e Biotecnologia. Documentos, 312; Embrapa Amazônia Oriental. Documentos, 378). http://www.infoteca.cnptia.embrapa.br/infoteca/handle/doc/913190.

Carvalho, A. V., Beckman, J. C., Maciel, R. de A. \& Farias Neto, J. T. de. (2013). Características físicas e químicas de frutos de pupunheira no Estado do Pará. Revista Brasileira de Fruticultura, 35 (3), 763-768. https://doi.org/10.1590/S0100-29452013000300013.

Clement, C. R., Kalil Filho, A. N., Modolo, V. A., Yuyama, K., Rodrigues, D. P., Leeuwen, J. van., Farias Neto, J. T. de., Cristo-Araújo, M. de. \& Flores, W. B. C. (2009). Domesticação e melhoramento de pupunha. In: Borém, A., Lopes, M. T. G. \& Clement, C. R. (Ed.). Domesticação e Melhoramento: Espécies Amazônicas. Editora UFV, 367-398.

Clement, C. R., Weber, J. C., Van Leeuwen, J., Domian, C. A., Cole, D. M., Lopez, L. A. A. \& Arguello, H. (2004). Why extensive research and development did not promote use of peach palm fruit in Latin America. Agroforestry Systems, 61 (1), 95-206. https://doi.org/10.1023/B:AGFO.0000028999.84655.17.

Clement, C. R. (1986). Descritores mínimos para el pejibaye (Bactris gasipaes H.B.K.) y sus implicaiones filogenética. Masters' Thesis- Escuela de Biologia, Universidad de Costa Rica, San José, Costa Rica. http://www.sidalc.net/cgi-bin/wxis.exe/?IsisScript=orton.xis\&method=post\&fo rmato $=2 \&$ cantidad $=1 \&$ expresion $=\mathrm{mfn}=053778$

Core Team R. (2014). R Core Team (2014). R: A language and environment for statistical computing. R Foundation for Statistical Computing. http://www.Rproject.org/. Acesso em: janeiro de 2019.

Cornelius, J. P., Weber, J. C., Sotelo-Montes, C. \& Ugarte-Guerra, L. J. (2010). Phenotypic correlations and site effects in a Peruvian landrace of peach palm (Bactris gasipaes Kunth). Euphytica, 173 (2), 173-183. https://link.springer.com/article/10.1007/s10681-009-0073-7.

Cristo-Araújo, M., Rodrigues, D. P., Astolfi-Filho, S. \& Clement, C. R. (2010). Genetic variability in the peach palm genebank with RAPD markers. Crop Breeding and Applied Biotechnology, 10 (3), 211-217. https://doi.org/10.1590/S1984-70332010000300005.

Farias Neto, J. T de, Yokomizo, G. K. I. \& Resende, M. D. V. de. (2020). Productive capacity and genect variation behavior in progênies from irrigated açaí according to plant age. Revista Agro@mbiente on-line, 14 (1), 1-14. http://dx.doi.org/10.18227/1982-8470ragro.v14i0.6409.

Farias Neto, J. T. d., Clement, R. C. \& Resende, M. D. V. de. (2013). Estimativas de parâmetros genéticos e ganho de seleção para produção de frutos em progênies de polinização aberta de pupunheira no Estado do Pará, Brasil. Bragantia, 72 (2), 122-126. https://doi.org/10.1590/S0006-87052013000200002.

Instituto Nacional de Metereologia (2019). Banco de dados meteorológico do INMET, Tomé-Açu. https://bdmep.inmet.gov.br/. 
Research, Society and Development, v. 10, n. 6, e25210615648, 2021

(CC BY 4.0) | ISSN 2525-3409 | DOI: http://dx.doi.org/10.33448/rsd-v10i6.15648

Kalil Filho, A. N., Clement, C. R., Resende, M. D. V. de., Farias Neto, J. T. de., Bergo, C. L., Yokomizo, G. K., Kaminski, P. E., Yuyama, K. \& Modolo, V. A. (2010). Programa de melhoramento genético de pupunha na Embrapa, IAC e Inpa. Colombo: Embrapa Florestas, 34p. (Embrapa Florestas. Documentos, 205). http://www.infoteca.cnptia.embrapa.br/infoteca/handle/doc/882495.

Mora Urpí, J. \& Clement, C. R. (1988). Races and populations of peach palm found in the Amazon basin. In: Clement, C. R. \& Coradin, L. (Ed.). Final report (revised): peach palm (Bactris gasipaes) germplasm bank. US-AID project report. Manaus: INPA e Embrapa Cenargen, 78-94. https://www.scielo.br/scielo.php?script=sci_nlinks\&ref=000124\&pid=S01020536199900030000300025\&lng=en .

Moraes, W. S., Santos, A. F. \& Fuzitani, E. J. (2017). Manejo inteligente de doenças da pupunheira. In: Rozane, D. E., Silva, C. A. \& Franchetti, M. (Org.). Palmito pupunha: do plantio a colheita. Registro: UNESP, Campus Experimental de Registro, 125-143.

Munsell Color. 1977. Munsell color charts for plant tissues. Macbeth Div. Kollmorgen Instruments Corp,

Oliveira, M. do S. P., Pinheiro, T. M. dos S. \& Fiala, M. A. (2019). Práticas para renovação do Banco de Germoplasma de espécie do gênero Euterpe (açaizeiros). Belém, PA: Embrapa Amazônia Oriental, p.10. (Comunicado técnico, 315) https://www.infoteca.cnptia.embrapa.br/infote ca/bitstream/doc/1113458/1/ComTec315.pdf.

Oliveira, M. do S. P., Sousa, T. S. \& Brandão, C. P. (2019). Divergência entre Indivíduos de Oenocarpus distichus Mart. (Bacaba-de-Leque) numa População de Belém, PA, por Meio de Caracteres Morfoagronômicos. Belém, PA: Embrapa Amazônia Oriental. p. 27. (Boletim de Pesquisa e Desenvolvimento, 136). https://www.infoteca.cnptia.embrapa.br/infoteca/bitstream/doc/1114208/1/BPD136.pdf.

Portal Alelo (2019). Dados de passaporte Banco Ativo Germoplasma de Pupunha. http://alelobag.cenargen.embrapa.br/AleloConsultas /Passaporte/detalhesBanco.do?idb=401.

Rodrigues, D. P., Astofil Filho, S. \& Clement, C. R. (2005). Molecular marker-mediated validation of morphologically defined landraces of pejibaye (Bactris gasipaes) and their phylogenetic relationships. Genetic Resources and Crop Evolution, 51 (8), 871-882. https://doi.org/10.1007/s10722-005-0774-2.

Santos, B. W. C. dos., Ferreira, F. M., Souza, V. F. de., Clement, C. R. \& Rocha, R. B. (2017). Análise discriminante das características físicas e químicas de frutos de pupunha (Bactris gasipaes Kunth) do alto Rio Madeira, Rondônia, Brasil. Revista Científica, Jaboticabal, 45 (2), $154-161$. http://dx.doi.org/10.15361/1984-5529.2017v45n2p154-161.

Silva, C. C. da., Rodrigues, D. P., Clement, C. R. \& Astolfi Filho, S. (2019). Molecular-genetic analysis for validation of peach palm (Bactris gasipaes Kunt) landraces using RAPD markers. Revista Científica, Jaboticabal, 47 (3), 313-320. http://dx.doi.org/10.15361/1984-5529.2019v47n3p313-320.

Sousa, N. R., Rodrigues, D. P., Clement, C. R., Nagao, E. O. \& Astofil Filho, S. (2001). Discriminação de raças primitivas de pupunha (Bactris gasipaes) na Amazônia brasileira por meio de marcadores moleculares (RAPDs). Acta Amazonica, 31 (4), 539-545. https://doi.org/10.1590/1809-43922001314545.

Vaz, A. B. (2010). Caracterização da interação Phytophthora palmivora x pupunheira quanto à patogênese, histopatologia e controle biológico. Dissertação (Mestrado em Produção Vegetal) - Universidade Estadual de Santa Cruz, Ilhéus, Bahia. http://nbcgib.uesc.br/ppgpv/pain el/paginas/uploads/ce12d2c0bddfa249c73b9468ea023a94.PDF. 